Project Number: 60451

Project Title:

\title{
Mechanics of Bubbles in Sludges and Slurries: Modeling Studies of Particulate Materials
}

This report covers only a portion of Project 60451, entitled "Mechanics of Bubbles in Sludges and Slurries," lead PI P. A. Gauglitz, Pacific Northwest National Laboratory; specifically that part dealing with modeling particulate materials.

Date: March 1, 2000

PI: William R. Rossen

Center for Petroleum and Geosystems Engineering

The University of Texas at Austin

Austin, TX 78712-1061

wrossen@pe.utexas.edu

Post-doctoral fellow: Dr. Seung Ihl Kam

\section{Specific DOE Problem Addressed}

Bubbles form within the slurry in waste tanks of the Hanford nuclear reservation by radiation-induced destruction of organics in solution and sub sequent diffusion of the products to bubble sites. If the fraction of gas in the slurry reduces slurry density below that of liquid above it, a "rollover event" occurs, in which potentially flammable quantities of gas are released into the vapor space at the top of the tank. It is therefore important to monitor the gas fraction in the slurry. One means of doing so is by tracking the response of liquid level in the tanks to changes in barometric pressure. Interpreting these data requires knowing the effective compressibility of bubbles in the slurry, which can be affected by capillary forces. In addition, it is desired to relate the mechanical properties of the slurry to the volume fractions of gas, solids and liquid in the slurry. This project examines the process of diffusive growth of bubbles within a porous medium and the volume response of this population to a change in barometric pressure. On a separate track, it examines the mechanical properties of dispersions of gas, solid and liquid, and the effect of capillary forces between bubbles and solids on the yield stress and compressibility of the slurry (modeled as a deformable periodic dispersion).

\section{Research Objectives:}

1) Model effective compressibility of bubbles dispersed in a rigid porous medium. Account for capillary forces on bubbles that restrain bubble response to ambient pressure changes, and therefore affect compressibility of bubbles. Account for diffusive growth process in context of strong capillary forces, that may bias distribution of bubble sizes in population and thereby affect effective compressibility.

2) Model yield stress and effective compressibility of a dispersion of gas, solids and liquid (assumed here to be deformable), focusing on capillary interactions between solid and bubbles, and effects of these interactions on mechanical properties. 


\section{Research Progress and Implications:}

With approximately 6 months remaining in this three-year project, we have determined the following:

Capillary forces affect the effective compressibility of individual bubbles in a rigid porous medium. Specifically, capillary forces reduce the volume response of most bubbles to a decrease in pressure, except for bubbles poised in a pore constriction, which may expand outwards into the next pore with a jump. Most of the volume response of a population of bubbles reflects the jumps of the fraction of this population poised at pore throats. There is a hysteresis in the volume response of bubbles, because bubbles that jump outwards upon a decrease in pressure do not in general jump back upon a subsequent equal increase in pressure.

Capillary forces also affect the initial distribution of bubbles in the pore space. As compressible bubbles grow by diffusion, capillary pressure rises as bubbles grow into pore throats. As a result, gas pressure and chemical potential rises, and the driving force for further diffusion to the bubble decreases. As a result, the distribution of bubbles is further distorted, favoring more bubbles poised just short of pore throats, ready to jump at a decrease in pressure.

The model fits the behavior of the Hanford tanks. Unfortunately, the fit to the data is not unique. Therefore, it is not possible to determine gas compressibility uniquely with our model without additional information on pore geometry.

A two-dimensional (2D) model for a regular array of solids, bubbles and liquid indicates that capillarity produces a yield stress in a bubbly slurry even in the absence of colloidal forces between the solids. The behavior is similar to that of two-dimensional foams without solids: as the material is sheared, bubbles are stretched, resisting the deformation, until a threshold displacement at which the bubbles and solids spontaneously rearrange themselves. The resistance to deformation gives rise to a yield stress for the material. As with foams with no solids, the wetter the slurry, the lower the yield stress, and for sufficiently wet slurries the yield stress disappears.

The compressibility of such a 2D slurry, in the absence of capillary effects, would be the product of gas compressibility and gas volume fraction. In extreme cases, with very fine solids and bubbles, the compressibility of the slurry is further reduced by capillary forces. There is a kink in the plot of compressibility as a function of volume fractions of solids and gas, which appears to be similar to a second-order phase transition.

\section{Planned Activities}

In the remainder of the project period, we plan to complete the modeling of bubble growth by diffusion and gas compressibility, further examine the fit to Hanford tank data, and write final reports and articles for publication on all these topics.

\section{Information Access}

Contact the PI at

wrossen@pe.utexas.edu

(512) 471-3246

fax (512) 471-9605

or Dr. Kam at

skam@magnolia.pe.utexas.edu 